\title{
ACCESSORY CHROMOSOMES IN POA ALPINA
}

\author{
ARNE MUNTZING \\ Institute of Genetics, University of Lund, Sweden
}

Received 20.ix.47

IN a strain of Poa alpina the chromosome conditions were found to be remarkable, a higher number of chromosomes being present at meiosis in the p.m.c. than in the root tips (Müntzing, 1946a).

Root tips were fixed from seedlings grown in small pots. Counts from this material showed that most of the plants had $2 n=14$, the remaining plants being triploid or approximately triploid. Preliminary studies of meiosis in 5 plants, found to have $2 n=14$ in the root tips, revealed the presence of more than 14 chromosomes in the p.m.c. of all these plants. In the best slide the p.m.c. had 19 chromosomes, the most frequent first metaphase associations of these chromosomes being $9_{1 \mathrm{I}}+\mathrm{I}_{\mathrm{I}}$ and $8_{\mathrm{II}}+3_{\mathrm{I}}$.

These preliminary observations have now been extended. Altogether somatic counts have been made in about 300 plants, representing three generations. Meiotic data are available from a total of 35 individuals.

Root tips were fixed in chrome-acetic-formalin after treatment with low temperature (about $o^{\circ} \mathrm{C}$.) the night before fixation. For meiosis fixation with chrome-acetic-formalin following pre-treatment with acetic alcohol gave very poor results, whereas Benda proved to be more satisfactory. Root tips as well as spikelets were sectioned and stained with crystal violet. Pollen mitosis may be studied with some difficulty, but so far only aceto-carmine gave satisfactory results. Attempts to stain with Feulgen were not successful.

\section{MEIOSIS IN THE POLLEN MOTHER CELLS}

The main result of these studies is the observation that accessory chromosomes ("B chromosomes") are present in every one of the 35 individuals examined. These bodies are smaller than the ordinary chromosomes ("A chromosomes") and, thus, represent a distinct category. In the material so far studied the number of B's was found to range from 2 to 8 .

As a rule the number of B's can be accurately counted only at I-A. Figs. I-7 represent some clear cases of this kind, the number of B's being 2, 4, 5, 6 and 8 respectively. In all these cases the smaller B's may be distinguished at first sight from the ordinary chromosomes, which show a regular disjunction in two groups of seven. 
The $\mathbf{B}$ chromosomes are also more spherical and do not show any sign of being composed by two chromatids. In the ordinary chromosomes, on the contrary, the normal chromatid split is frequently visible.
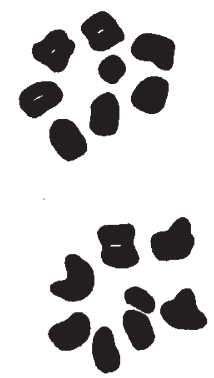

Fig. I : two B.
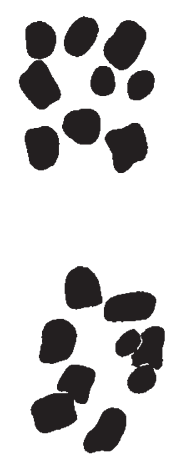

Fig. 2 : four B.
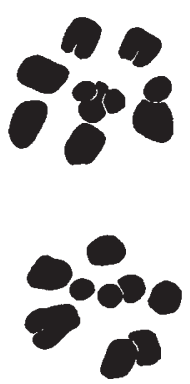

Frg. 3 : five $\mathrm{B}$.
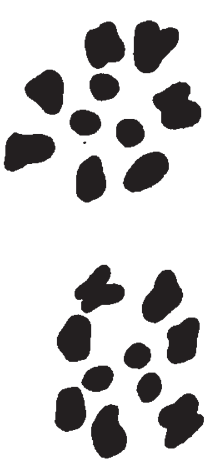

FIg. 4 : $\operatorname{six}$ B.
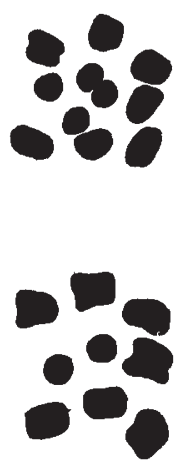

Fig. 5 : $\operatorname{six} B$.
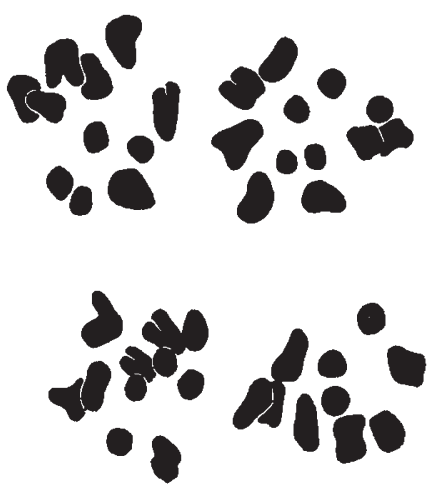

FIG. 6 : eight B. FIG. 7 : eight B.

Figs. 1-7.-First anaphase distributions from various plants, representing different numbers of $\mathrm{B}$ chromosomes in addition to the $14 \mathrm{~A}$ chromosomes. Fig. I : two B chromosomes, one in each plate; fig. 2, four B chromosomes, two in each plate; fig. 3 , five B chromosomes, the cell representing the distribution $7 \mathrm{~A}+2 \mathrm{~B}-3 \mathrm{~B}+7 \mathrm{~A}$; figs. $4-5$, six $\mathrm{B}$ chromosomes, in fig. 4 distributed as $3-3$, in fig. 5 as $2-4$; figs. 6-7, eight $\mathrm{B}$ chromosomes, in fig. 6 distributed as $4-4$, in fig. 7 as $3-5$.

In the following table the data on the number of accessory chromosomes are summarised.

\begin{tabular}{|c|c|c|c|c|c|c|c|c|}
\hline & \multicolumn{8}{|c|}{ Number of chromosomes } \\
\hline & $14+2$ & $14+3$ & $14+4$ & $14+5$ & $14+6$ & $14+7$ & $14+8$ & $n$ \\
\hline Accurate counts & 2 & $\mathbf{I}$ & 5 & 2 & 4 & $\ldots$ & 2 & I6 \\
\hline Approximate counts & $\mathbf{I}$ & $\mathbf{I}$ & 6 & 2 & 5 & $\mathbf{I}$ & 2 & 18 \\
\hline Total . & 3 & 2 & I I & 4 & 9 & I & 4 & 34 \\
\hline
\end{tabular}


In 16 cases the counts were accurate, in 18 cases they were not quite reliable. These approximate counts, however, show a quite similar distribution to the accurate ones and probably reflect the true situation. The 34 values given in the table represent 26 different individuals, more than one chromosome number being observed in six plants. In seven other plants fixation was bad, but it was clearly observed that accessory chromosomes were present. Two plants, finally, were approximately triploid as regards the normal chromosomes and were also found to have accessory chromosomes in the p.m.c.

In the table there is an obvious preponderance of even numbers of $\mathrm{B}$ chromosomes. In the series representing the accurate counts there are 13 even and 3 odd values, the corresponding ratio in the \pm series being $14: 4$. According to a $\chi^{2}$ test the probability that I $3: 3$ represents a $\mathrm{I}:$ I ratio is intermediate between 0.02 and 0.01 . The corresponding $P$ of the ratio $14: 4$ has the same value. The ratio for the total series will be $27: 7$, giving a $\chi^{2}$ of I I 76 and a $P$ smaller than 0.001 . Thus, there can be no doubt that in this material even numbers of accessory chromosomes are more frequent than odd numbers.

Chromosome pairing was studied at diakinesis and first metaphase. The B's were never observed to pair with the normal chromosomes, and when more than two B's are present no larger associations than bivalents seem to occur. In the preliminary paper (loc. cit.) trivalents were reported to be present in some cells. This statement was based on observations of I-M plates in polar view, such as figs. 8-9. These figures are from a plant (I. 20) with 5 B's (the same as is represented by fig. 3). In figs. 8 and 9 the configurations seem to be $\mathrm{I}_{\mathrm{III}}+8_{\mathrm{II}}$ and $I_{I I I}+7_{I I}+2{ }_{I}$. However, a detailed study of I-M groups in side view had convinced me that trivalents seldom or never occur in this material. Hence the apparent trivalents in these cells probably represent a bivalent and a univalent lying close together.

Figs. Io and I I represent I-M groups in side view from the same fixation. In both cells the configuration is $8 \mathrm{II}+3 \mathrm{I}$. In Fig. Io the B's are present as $2{ }_{I I}+I_{I}$, in fig. II as $I_{I I}+3 I$. Thus, in fig. Io two of the univalents are A univalents, whereas in fig. I I all the univalents are probably $\mathrm{B}$ univalents.

In fig. I 2 the pairing conditions are specially simple, 8 bivalents being present. One of these bivalents is smaller than the other ones and certainly represents the two extra B chromosomes of this plant. The I-A group in fig. I is from the same plant.

Figs. I 3 and I 4 show I-M groups from a plant (2I6-I) with 6 B's. In fig. I 4 with $1^{1}{ }_{I I}$ all the chromosomes are members of bivalents. The second and third from the left are certainly formed by B's. The identity of the third B bivalent is less certain. In fig. I 3 the configuration is $7 \mathrm{II}+6$. Judging from the size differences the $A$ chromosomes are present as $6_{\mathrm{II}}+2_{\mathrm{I}}$, the $\mathrm{B}$ chromosomes as $\mathrm{I}_{\mathrm{II}}+4 \mathrm{I}$. 


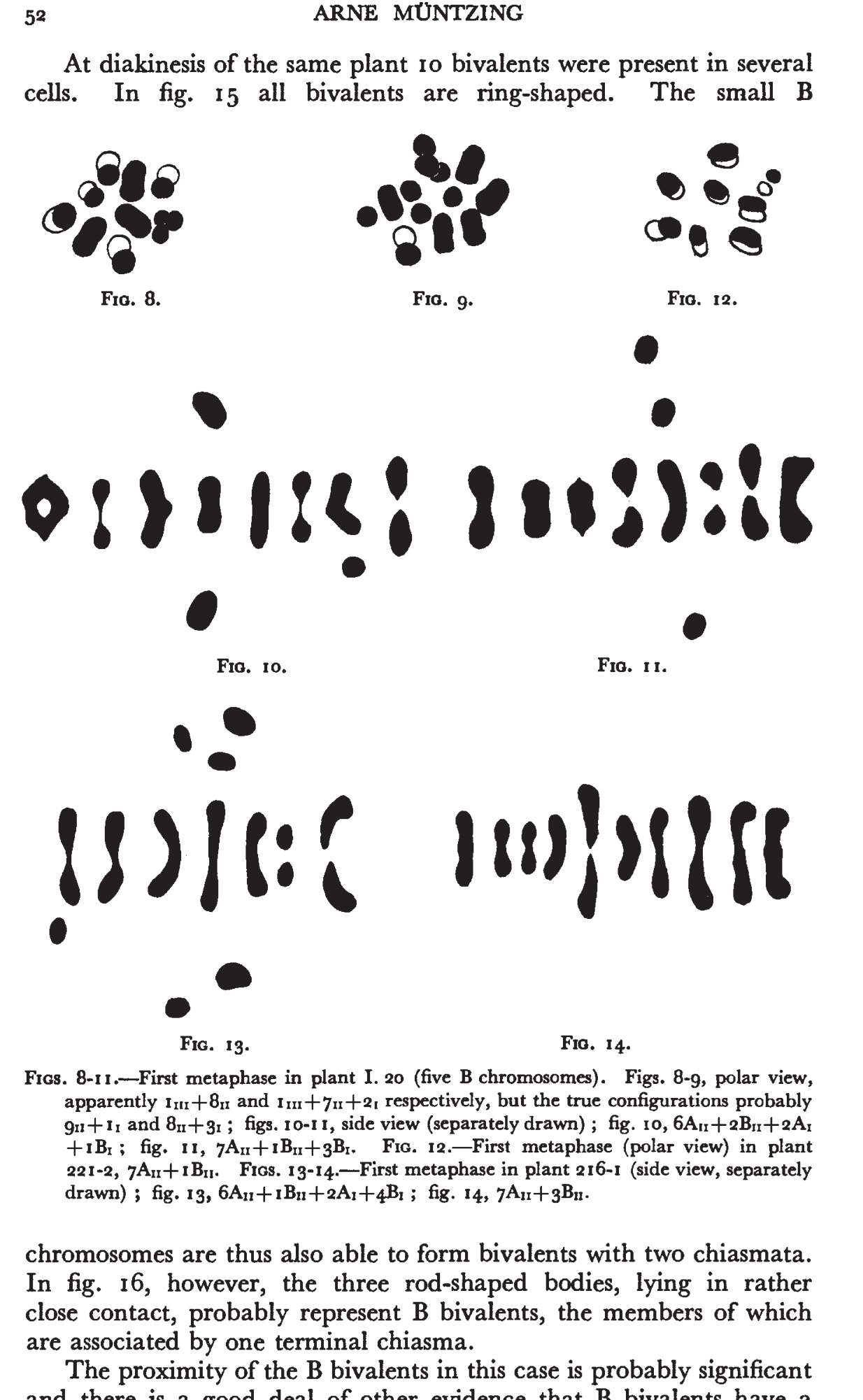


tendency to stick together. This is evident from figs. 17 and 18 , representing diakinesis in another plant (I. 19). In fig. I7 there are 7 normal bivalents and a peculiar structure similar to a quadrivalent. From other cells of the same slide it is clear, however, that the apparent quadrivalent and similar structures in other cells (cf. fig. 18) represent two B bivalents which are loosely attached to each other, owing to a certain degree of stickiness of the B's. In this particular slide the colour of the B's at diakinesis appeared to be different from that of the A's, thus facilitating the identification of the B bivalents. This difference in shade, however, was very small and was not observed in slides from other plants, representing the same stage.
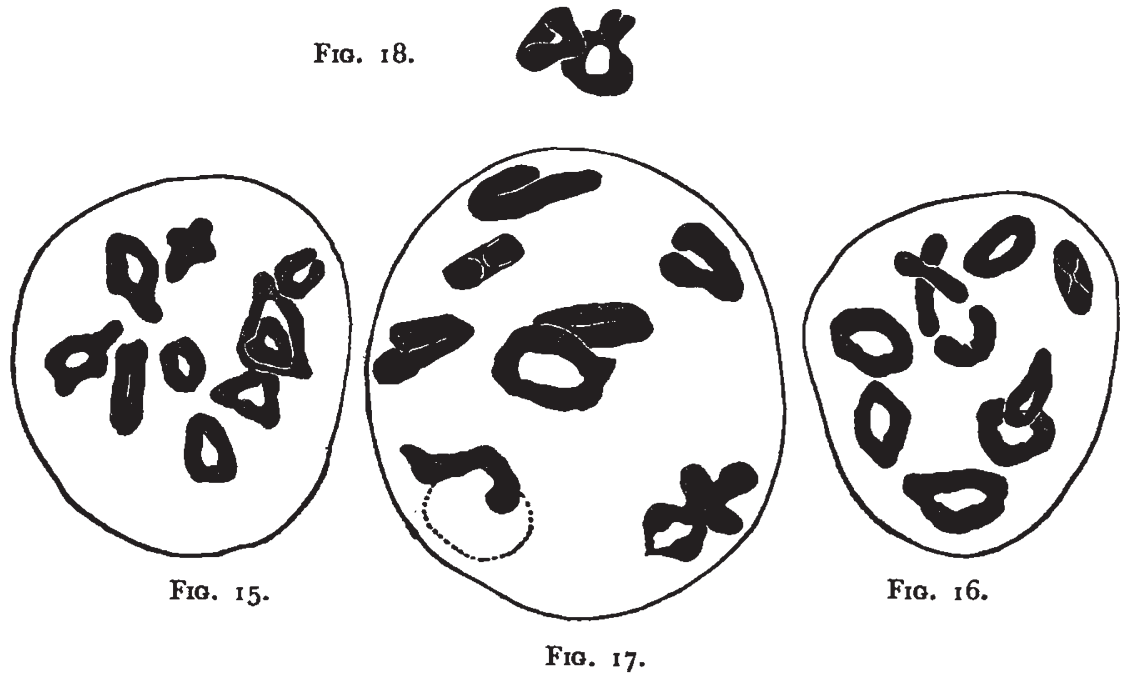

Figs. $15-16$.-Diakinesis in plant $216-1$, 1o bivalents. FrGs. 17-18.-Diakinesis in plant $I$. 19 ; fig. $17,7 A_{11}+{ }_{2} B_{11}$, the latter sticking together : fig. 18 , two $B$ bivalents sticking together.

The tendency of B bivalents to lie together is rather marked at first metaphase, thus hindering the analysis of I-M plates in side views. When two such small bivalents are in intimate contact it is often difficult to decide if such a structure represents two or four chromosomes. The secondary association of $\mathrm{B}$ bivalents at diakinesis and I-M may also be traced at first anaphase. In I-A groups containing more than one B, the B's are frequently in a more or less close proximity to each other ( $f f$. figs. 2-7).

The study of diakinesis revealed that the B's are not satellite chromosomes and are not associated with the nucleolus ( $c f$. fig. I7). In figs. 15 and 16 the nucleolus had not taken the stain enough to be discernible, but in several other cases, besides that represented by fig. I7, it was clear that the B's were not involved in the formation of the nucleolus. 
At I-A the B chromosomes pass to the poles without division, not only if they have been members of bivalents but also if they have been unpaired. Though unpaired B's are frequent at I-M in some plants they were never seen to divide at first anaphase. This is in a marked contrast to the A chromosomes, which in triploids or aneuploids of the same strain usually divide at I-A. The undivided B's are in most cases included in the interphase nuclei and, thus, micronuclei at this stage are rather rare. In plants I. I4 and 223-3, counts of univalents at I-M, and of micronuclei at interphase, were undertaken; the following distributions were obtained :-

\begin{tabular}{|c|c|c|c|c|c|c|c|c|c|c|}
\hline \multicolumn{4}{|c|}{ Number of univalents or micronuclei } & & & & 2 & 3 & 4 & $n$ \\
\hline " & $\eta$ & cell & in I. 14 ; metaphase I & & & & 4 & 4 & 3 & Io6 \\
\hline " & " & , & int & & 9 & & & & & 100 \\
\hline ", & " & , & „, 223-3, metaphase I & & 2 & & 2 & 3 & & 40 \\
\hline , & " & , & „223-3, interphase & & $5 s$ & & & & & \\
\hline
\end{tabular}

In I. 14 the number of B's is 6 and in 223-3 it is 8. Though the numbers in both cases are even, a good deal of non-pairing evidently occurs. In a few cases A chromosomes may be involved, but most of the unpaired chromosomes are certainly B's. According to the values given above, about half of the cells contained a variable number of univalents at I-M but only a few per cent. of the cells at interphase contained micronuclei. This is true of both the plants examined and other less detailed data indicate that this is the general type of behaviour of the B's in this strain.

The occurrence of $B$ univalents at I-M accounts for the uneven distribution of B's at I-A observed in several cases. Thus in fig. 5 the distribution is $4-2$ instead of the normal 3-3 (fig. 4), and in figs. 6 and 7 two I-A distributions from the same slide are 4-4 and 5-3.

No detailed data from the second division and the tetrads are as yet available, but the preliminary observations made indicate that these stages are rather regular.

A few observations from the pollen mitosis may also be mentioned. Figs. 19 and 20 show a metaphase and a commencing anaphase of the first pollen mitosis. These pictures are from plant I. I9, having at least four B's. Though the pictures were drawn before meiosis had been studied and the size difference between the $\mathrm{A}$ and $\mathrm{B}$ chromosomes had been realised, the two categories of chromosomes may be rather well distinguished. Thus in fig. I9 the three B's are situated near the satellite chromosome, and in fig. 20 the B's are probably represented by the second, fourth and seventh pair from the right. In these pairs the chromatids seem to have separated somewhat earlier than in most of the other chromosomes. Thus division of the B's is not delayed and there is no indication of non-disjunction. Anaphases of the first pollen mitosis have also been observed in some other plants of this strain, carrying B's. These anaphases were always found to be 
regular. Thus in this material the first pollen mitosis shows no special complications.

The number of accessory chromosomes is fairly constant in the p.m.c. of each individual. Evidence has also been obtained, however, that this number may vary. The following cases may be mentioned :-

Plant I. 20. Two fixations were made, one in 1944 , one in 1945. As already mentioned (loc. cit.) the first fixation contained p.m.c. with 5 B's ( $c f$. fig. 3 ), whereas in the other fixation there was one B less. This difference is quite certain and based on a number of good first anaphases of both fixations.

Plant I. I9. One fixation from I944 showed 4 B's at diakinesis (fig. I7). In another fixation made in I945 good I-M's and I-A's

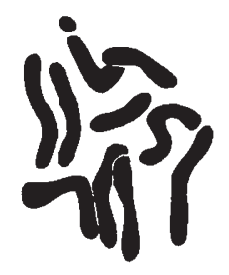

Fig. 19.

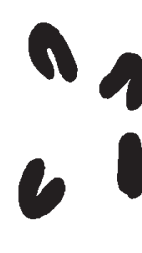

FIGs. 19-20.-First pollen mitosis in plant I. 19 ; fig. 19, metaphase $7 \mathrm{~A}+{ }_{3} \mathrm{~B}$; fig. 12, commencing anaphase, to chromosomes dividing.

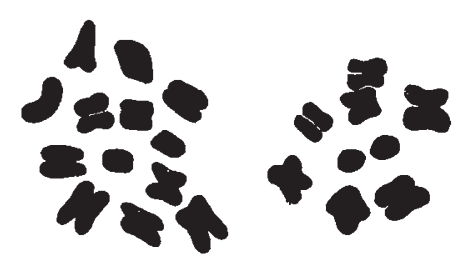

FIG. 21. - First anaphase in plant $\mathrm{S} .023(18 \mathrm{~A}+4 \mathrm{~B})$, showing the distribution $1 \mathrm{~A}+2 \mathrm{~B}-7 \mathrm{~A}+2 \mathrm{~B}$.

were studied, the total chromosome number ranging from 18 to 20 . Two quite clear I-A groups in the same section differed in chromosome number, one showing the distribution 9-9, the other one 9-Io. Thus the number of B's in this plant probably ranged from 4 to 6 .

Plant 208-6. In one fixation from 1946 there were clearly $14+6$ chromosomes in several cells at diakinesis and I-A. In another panicle fixed the same day good I-A's revealed the presence of 8 B's.

Plant 215-I. In this plant the data are less complete, but in one fixation I-M's in side view showed $8_{\mathrm{II}}$; in another fixation from the same day, cells at diakinesis and I-M (polar view) also showed $8_{\mathrm{II}}$, the smaller B bivalent being clearly seen at I-M. Another good I-M plate in polar view had 7 normal bivalents +2 small B bivalents.

From the data given above it is evident that the number of B chromosomes in the p.m.c. may vary within the individual, not 
only in different years and in different panicles but also within the same anther loculus. In order to study this phenomenon more closely, clones have been made of several individuals. Meiotic studies of the different clone plants will show the extent of the intra-individual variation.

\section{THE SOMATIC CHROMOSOMES}

A typical somatic plate from a root tip is represented by fig. 22. All the 14 chromosomes have median or sub-median constrictions. One chromosome pair (probably the SAT chromosome) differs from the others by having a sub-median achromatic region. In fig. 22 this region is rather short; in fig. 23 it seems to be much longer,

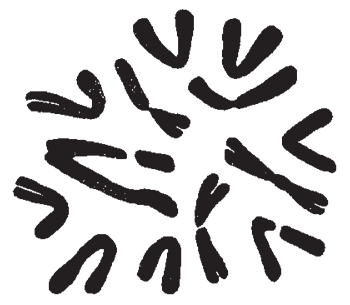

FIG. 22.

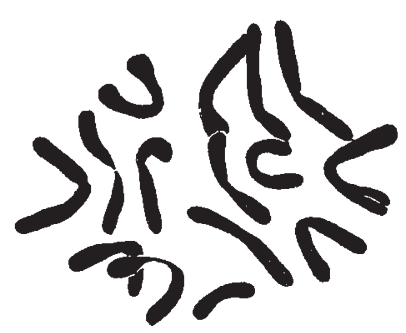

Fro. 24.

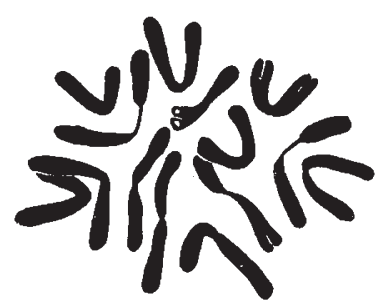

Fio. 23.

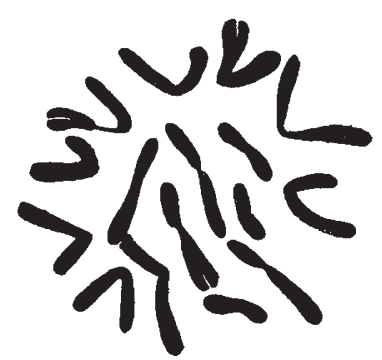

Fig. 25.

Figs. 22-25.- Somatic plates from root tips. Figs. $22-23,2 n=14$. In fig. 23 the achromatic region in one pair is more marked than in fig. 22. Fig. 24, one B chromosome in addition to the $14 \mathrm{~A}$ chromosomes; fig. $25,3^{\mathrm{B}}+{ }_{13} \mathrm{~A}$ or, possibly, $2 \mathrm{~B}+14 \mathrm{~A}$.

the two arms of the chromosomes in question lying rather far apart and apparently being connected by a thin thread. In this plate it is also obvious that the shorter arm of this chromosome is heterochromatic, being somewhat broader and more deeply stained than the longer arm. In extreme cases the two arms may be so far apart that they appear to be quite separate.

Fig. 24 is an exceptional root tip plate, showing one B chromosome in addition to the ${ }_{14}$ A's. Fig. 25 is still more deviating, the chromosome complement apparently consisting of 3 B's + I $_{3}$ A's. The absence of one $\mathrm{A}$ is remarkable, but it is not quite excluded that one of the apparent B's is really an A which is more contracted than usual. In both plates the B's differ from the A's by their smaller size. No 
difference in stain intensity between A's and B's could be seen in this and other similar cells. The B's evidently have median centromeres as was also indicated by the pollen mitosis (figs. I9 and 20).

The plates represented by figs. 24 and 25 are from plant 208-6, which at meiosis was found to have 6 or 8 B's. Apart from figs. 24 and 25, fifteen other plates of the same slide were studied. These plates together, from three different root tips, showed the following variation in chromosome number :-

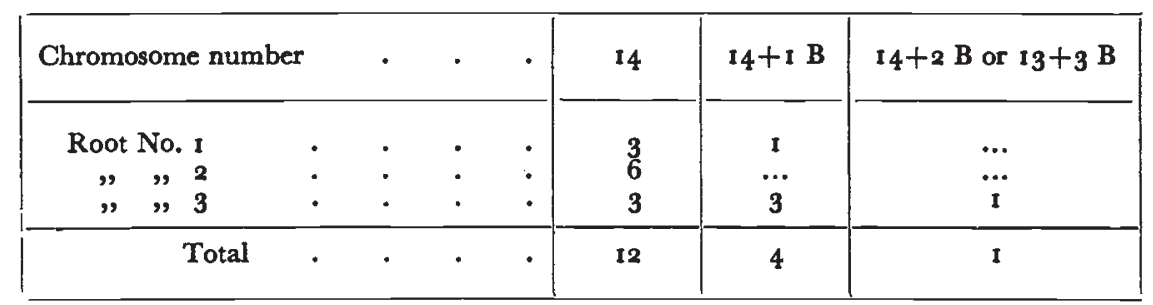

Thus twelve of the seventeen plates had no B's, in five plates there were I, 2 or possibly 3 B's and, of these five deviating plates, four were found in Root No. 3. Elimination of the B's is thus almost complete but especially in one of the roots a few B's are still present.

Among the other 34 individuals, in which meiosis was studied, somatic counts in root tips were made in 30. As usual the root tips were gathered from young seedlings, growing in small pots. In 27 of these plants only I 4 chromosomes were observed, in one plant two additional small chromosomes were present in one plate (this plant had \pm 6 B's at meiosis). One plant was a true triploid with about 2 I chromosomes in the root tips. And one plant finally appeared to be exceptional by having I 7 or I 8 chromosomes. Meiotic studies in this plant revealed, however, that it was a true aneuploid, having I 8 A's and 4 B's. This is evident from fig. $2 \mathrm{I}$, showing the I-A distribution of I I $\mathrm{A}+2 \mathrm{~B}$ from $7 \mathrm{~A}+2 \mathrm{~B}$.

The root tip counts in these 30 plants were less detailed than in plant 208-6, and it is probable that a more extensive work would have revealed occasional plates with one or a few $B$ chromosomes. Nevertheless, it is obvious that 14 is the characteristic and quite predominating chromosome number, showing that as a rule the $B$ chromosomes are entirely eliminated from these parts of the plant.

This conclusion is based not only on somatic counts in individuals also studied with regard to meiosis, but rests on a much larger material. Altogether somatic counts have so far been made in about 300 individuals of this strain, and with a small number of exceptions these individuals had only 14 chromosomes in the root tips. Judging from the meiotic results, accessory chromosomes must occur at meiosis in most or all of these plants, though they are absent in the root tips.

The exact time and place of elimination of the $\mathbf{B}$ chromosomes in 
this material is not yet known. My colleague, $\operatorname{Dr} \mathrm{A}$. Håkansson tells me, however, that he observed the B's in the embryos. He also found many in the main root fixed from germinating seeds. Thus, according to these preliminary data, elimination of the B's occurs at a relatively late stage This is in accordance with the fact reported above, that occasionally one or a few B chromosomes may still occur in the root tips of young seedling plants growing in pots.

\section{DISCUSSION}

Accessory chromosomes have already been reported in many species of plants and animals and the list of such cases is steadily increasing. References to the literature in question may be found in the recent papers by Håkansson (1945), Müntzing (1946), Östergren (1947) and, as regards animal species, in the textbook of White (1945).

Accessory chromosomes certainly represent a heterogeneous assemblage. Nevertheless the cases best studied have several features in common, thus allowing a characterisation of these peculiar chromosomes. As a rule the $B$ chromosomes are smaller than the A chromosomes, they are often heterochromatic and show no homology to the A's, they have slightly deleterious genetical effects but are much less active than the A's. A very striking peculiarity is their numerical increase by non-disjunction at the pollen mitosis and, in rye, at the corresponding stage on the female side. In rye and probably also in Anthoxanthum the accumulation by means of non-disjunction is counterbalanced by the deleterious effects of the B's on fertility and vigour, only a small fraction of plants in the population carrying them.

The B chromosomes in the present material of Poa alpina are typical in some respects but deviate in others. The most striking feature is the regular occurrence of B's in varying numbers in the p.m.c. and their absence in the root tips. Thus they are very well preserved in the "germ line" but eliminated from other parts of the plants. The only case of this kind previously observed in a plant species is that described by Janaki-Ammal (I940) and Darlington and Thomas (194I) in Sorghum purpureo-sericeum. Among Ioo plants of this species Janaki-Ammal found 40 to have from I to 6 extra chromosomes. These chromosomes were never found in the roots but were confined to the shoot system. Thus B's in this material are frequent, but plants without them were in a majority.

In my Poa material all 35 plants examined were found to have B's. Since they occur in every plant they might be regarded as normal members of the chromosome complement. This is excluded because their number is variable and so far ranging from 2 to 8 , the most frequent numbers being 4 and 6. Moreover, their genetical effects must be none or very slight, the plants with many B's apparently not differing in vigour and fertility from those having few B's. This, 
however, must be studied in more detail later on, especially if it is possible by selection to obtain plants free from B's. At any rate, plants with 6 or 8 of them have normal vigour and a good pollen fertility.

A very interesting fact is the predominance of even numbers of B's in the p.m.c., plants or cells with odd numbers being significantly less frequent. The mechanism responsible for the preponderance of even numbers is not known. As a working hypothesis it may be suggested that the cause is somatic non-disjunction, usually involving all of them simultaneously and not only single B's. Doubling will then always give an even number.

Such somatic non-disjunction might also account for the absence of the B's in the roots. In rye there is a directed non-disjunction in the p.m.c. and embryo-sacs (Müntzing, 1945, 1946b) and similar phenomena have also been observed in other plant species with B's. If, in the present material, the postulated somatic non-disjunction leads to the B chromatids preferably passing to the "germ line" pole, the cells giving rise to the roots will be free from B's. At the same time this non-disjunction would lead to somatic accumulation, increasing the number of B's in the p.m.c. and probably also in the embryo-sacs ( $c f$. Östergren, 1947, p. 290).

In rye and some other plants with $B$ 's this non-disjunction is accomplished at the first or second pollen mitosis and in rye also at a corresponding stage on the female side. The first pollen mitosis in the present material of Poa alpina clearly shows that there is no nondisjunction of the B's at this stage. The high frequency of B's in this strain, however, indicates that some other mechanism of reduplication is at work. Judging from the data so far available it is probable that this reduplication is premeiotic rather than postmeiotic. Somatic irregularities are indeed shown by the cases reported above, in which different parts of the same individual differed in the number of B's.

By subjecting roots of Secale to a temperature of $0^{\circ} \mathrm{C}$. for 13 days, somatic non-disjunction of $\mathrm{B}$ 's could be induced ( $c f$. Darlington, 1947). It will be interesting to examine whether the B elimination in Poa alpina may also be influenced by changes in temperature.

The meiotic elimination of the B's in Poa is very slight owing to their non-division at I-A. In this respect they are similar to the B's in Anthoxanthum (Ostergren, 1947) and clearly differ from the A's, which usually divide at I-A if they have been unpaired. This differential behaviour was best seen in a hybrid between the sexual diploid strain and a Scandinavian apomict which will be described later.

In the present material the degree of non-conjunction of the B's was found to differ considerably from plant to plant. Usually, however, these chromosomes are represented by bivalents at diakinesis and I-M. They were never seen to pair with the A's and in this respect they behave as typical B's. A peculiar feature is their 
apparent inability to form larger associations than bivalents. Chiasma counts could not be made, but at diakinesis the B bivalents were often ring-shaped, and thus, from a mechanical point of view, B multivalents ought to be formed. The frequent secondary association of two or more $\mathrm{B}$ bivalents at diakinesis and I-M and the resulting proximity of the B's at I-A is at least secondarily favoured by the obvious stickiness of the B's. This is in good accordance with the findings of Thomas and Revell (I946) in Cicer arietinum. In this species there were pachytene fusions between highly charged heterochromatic regions from different bivalents and this pairing was thought to be responsible for the secondary association at metaphase.

The B's in Poa are probably also heterochromatic to some extent. The stickiness is one symptom and the rounded shape and smooth outline at I-M and I-A another. In one slide, containing cells at diakinesis, the $\mathrm{B}$ bivalents showed a very slight but noticeable difference in staining reaction in comparison with the A chromosomes.

Among the A's one pair with a long achromatic region was found to be heterochromatic in the short arm, this arm being thicker and more intensively stained in root tip metaphases than the other arm and the other chromosomes. The B's might have arisen from this heterochromatic chromosome arm, especially since the connection between this arm and the rest of the chromosome appears to be rather weak. This view, however, is contradicted by the fact that the B's have median centromeres and do not pair with this chromosome arm at meiosis. Thus the origin as well as the function of the accessory chromosomes in Poa alpina is still obscure.

\section{SUMMARY}

( $\mathrm{I}$ ) Meiosis was studied in 35 individuals of a diploid sexual strain of Poa alpina. In all of these plants accessory or B chromosomes were present in the p.m.c. These bodies are smaller than the 14 ordinary chromosomes and represent a distinct category, showing no homology to the A chromosomes. In the material so far studied the number of B chromosomes ranged from 2 to 8 .

(2) When more than two B's were present at diakinesis and first metaphase no larger associations than bivalents were observed. Different B bivalents have a tendency to stick together, and the B chromosomes also show other symptoms of being heterochromatic.

(3) In contrast to A univalents, unpaired B's do not divide at first anaphase and are seldom eliminated. The first pollen mitosis is regular, the B's showing no tendency to non-disjunction.

(4) In the p.m.c. the number of accessory chromosomes is fairly constant, but this number may sometimes vary within the individual, not only in different years and in different panicles but also within the same anther loculus.

(5) As a rule the $\mathbf{B}$ chromosomes are entirely eliminated from 
the root tips of seedlings and older plants, only the 14 A's being present. In exceptional cases one or a few B's may be present. They are smaller than the A's and have a median centromere.

(6) In the p.m.c. even numbers of B's are more frequent than odd numbers. Tentatively this fact is explained by the assumption of somatic non-disjunction usually involving all the B's simultaneously. If the B-chromatids preferably pass to the "germ line" pole, the cells giving rise to the roots will be free from B chromosomes. At the same time this non-disjunction would represent a somatic accumulation mechanism, tending to increase the number of $B$ chromosomes in the p.m.c. and embryo-sacs.

\section{REFERENCES}

DARLINGTON, C. D. 1947.

John Innes Hort. Inst.

37th Ann. Rep.

DARLINGTON, G. D., AND THOMAS, P. T. 1941.

Morbid mitosis and the activity of inert chromosomes in Sorghum.

P.R.S. B. $130,127-150$.

HÅKANSSON, A. 1945 .

Úberzählige Chromosomen in einer Rasse von Godetia nutans Hiorth.

Bot. Not. I 945, pp. I-1 9 .

JANAKI-AMMAL, E. $\mathrm{K}$. 1940 .

Chromosome diminution in a plant.

Nature 146,839 .

MÜNTZING, A. 1945 .

Cytological studies of extra fragment chromosomes in rye. II. Transmission and multiplication of standard fragments and iso-fragments.

Hereditas 3I, 457-477.

MÜNTZING, A. $1946 a$.

Different chromosome numbers in root tips and pollen mother cells in a sexual strain of Poa alpina.

Hereditas 32, 127-1 29.

MÜNTZING, A. $1946 b$.

Cytological studies of extra fragment chromosomes in rye. III. The mechanism of non-disjunction at the pollen mitosis.

Hereditas 32, 97-1 I9.

THOMAS, P. T., AND REVELL, S. H. 1946 .

Secondary association and heterochromatic attraction. I. Cicer arietinum.

Ann. Bot. 9, I 59-164.

WHITE, M. J. D. 1945 .

Animal cytology and evolution. London.

ÖSTERGREN, G. 1947 .

Heterochromatic B chromosomes in Anthoxanthum.

Hereditas 33, $26 \mathrm{I}-296$. 